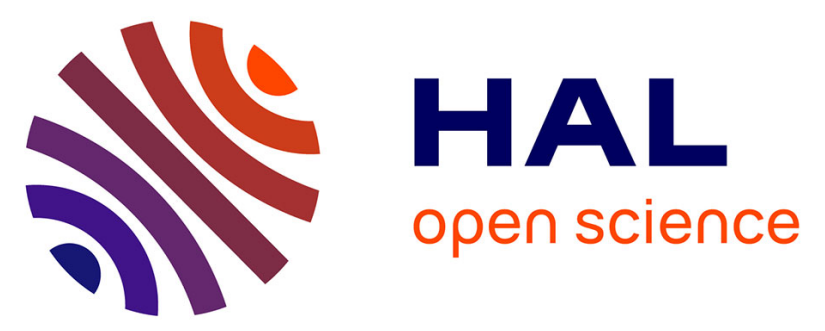

\title{
Antioxidant effects of extra virgin olive oil enriched by myrtle phenolic extracts on iron-mediated lipid peroxidation under intestinal conditions model
}

Sofiane Dairi, Marie-Annette Carbonneau, Teresa Galeano-Diaz, Hocine Remini, Farid Dahmoune, Omar Aoun, Amine Belbahi, Céline Lauret, Jean-Paul Cristol, Khodir Madani

\section{To cite this version:}

Sofiane Dairi, Marie-Annette Carbonneau, Teresa Galeano-Diaz, Hocine Remini, Farid Dahmoune, et al.. Antioxidant effects of extra virgin olive oil enriched by myrtle phenolic extracts on ironmediated lipid peroxidation under intestinal conditions model. Food Chemistry, 2017, 237, pp.297 304. 10.1016/j.foodchem.2017.04.106 . hal-01757133

\section{HAL Id: hal-01757133 \\ https://hal.science/hal-01757133}

Submitted on 14 Dec 2019

HAL is a multi-disciplinary open access archive for the deposit and dissemination of scientific research documents, whether they are published or not. The documents may come from teaching and research institutions in France or abroad, or from public or private research centers.
L'archive ouverte pluridisciplinaire $\mathbf{H A L}$, est destinée au dépôt et à la diffusion de documents scientifiques de niveau recherche, publiés ou non, émanant des établissements d'enseignement et de recherche français ou étrangers, des laboratoires publics ou privés. 


\section{Antioxidant effects of extra virgin olive oil enriched by myrtle phenolic extracts on iron-mediated lipid peroxidation under intestinal conditions model}

Sofiane Dairi ${ }^{\mathrm{a}, \mathrm{b}}$, Marie-Annette Carbonneau ${ }^{\mathrm{c}}$, Teresa Galeano-Diaz ${ }^{\mathrm{d}}$, Hocine Remini ${ }^{\mathrm{a}, \mathrm{e}}$, Farid Dahmoune ${ }^{\mathrm{a}, \mathrm{e}, *}$, Omar Aoun ${ }^{\mathrm{a}, \mathrm{f}}$, Amine Belbahi $^{\mathrm{a}}$, Céline Lauret ${ }^{\mathrm{c}}$, Jean-Paul Cristol ${ }^{\mathrm{c}}$, Khodir Madani ${ }^{\mathrm{a}}$

${ }^{a}$ Laboratoire Biomathématiques, Biophysique, Biochimie et de Scientométrie (L3BS), Faculté des Sciences de la Nature et de la Vie, Université de Bejaia, 06000 Bejaia, Algeria

${ }^{\mathrm{b}}$ Département de Microbiologie Appliquée et Sciences Alimentaires, Faculté des Sciences de la Nature et de la Vie, Université de Jijel, 18000 Jijel, Algeria

${ }^{\mathrm{C}}$ UMR 204 NUTRIPASS, University Institute of Clinical Research - 641, Av. Doyen Gaston Giraud, 34093 Montpellier Cedex5, France

${ }^{\mathrm{d}}$ University of Extremadura, Analytical Chemistry Department, Badajoz, Spain

${ }^{e}$ Département de Biologie, Faculté des Sciences de la Nature et de la Vie et des Sciences de la Terre, Université de Bouira, 10000 Bouira, Algeria

${ }^{\mathrm{f}}$ Département de Biologie, Faculté des Sciences de la Nature et de la Vie et des Sciences de la Terre, Université de Khemis Miliana, 44225 Khemis Miliana, Algeria

Keywords:

Myrtus communis

Lipid dispersion

Antioxidant activity

Functional food

Iron (III)/ascorbic acid system

\section{A B S T R A C T}

Chelating and free radicals scavenging activities of extra virgin olive oil (EVOO) enriched by Myrtus communis phenolic compounds (McPCs), $\alpha$-tocopherol and Butylated hydroxytoluene (BHT) were evaluated using chemical assays, 2,2-diphenyl-1-picrylhydrazyl (DPPH) and Oxygen radical absorbance capacity (ORAC), and biological model as 2,2'-azobis (2-aminopropane) dihydrochloride (AAPH) or Fe ${ }^{+3} /$ Ascorbic acid $\left(\mathrm{Fe}^{+3} / \mathrm{AsA}\right)$ system mediated peroxidation of $\mathrm{L}-\alpha$-phosphatidylcholine aqueous dispersions stabilized by bile salts (BS) under simulated intestinal conditions ( $\mathrm{pH} 7.4$ ). McPC-EEVOO increased significantly the neutralization of DPPH radical and AAPH-derived radicals in ORAC assay more than $\alpha$-tocopherol and BHT. The phospholipid stability increased by a factor of $33.6 \%, 34.8 \%, 19.3 \%$ and $10.7 \%$ for myrtle microwave assisted extraction (MAE) and conventional extraction (CE) extracts, $\boldsymbol{\alpha}$-tocopherol and BHT, respectively, as compared to the control (EVOO without enrichment) in $\mathrm{Fe}^{+3} /$ AsA system. But a slightly additive effect was observed when AAPH system was used. Our observation showed that McPCs may interact positively with EVOO to inhibit phospholipid peroxidation, and thus, McPC-EEVOO could be a potential functional food.

\section{Introduction}

Lipid oxidation has been shown to be associated with the disturbance of the structure and the function loss of the cell membranes and the oxidative modification of low-density lipoproteins and is an important initial event for the pathogenesis of atherosclerosis (Marinova, Toneva, \& Yanishlieva, 2008). Data related to the oxidative fate of lipids during digestion remain relatively scarce. The acidic gastric environment, combined with the presence of

Abbreviations: McPC, Myrtus communis phenolic compound; EVOO, extra virgin olive oil; EEVOO, enriched extra virgin olive oil; MAE, microwave assisted extraction; CE, conventional extraction; EYPC, egg yolk phosphatidylcholine; AUC, area under curve; M. com, Myrtus communis.

* Corresponding author at: Département de Biologie, Faculté des Sciences de la Nature et de la Vie et des Sciences de la Terre, Université de Bouira, 10000 Bouira, Algeria.

E-mail address: farid.dahmoune@univ-bejaia.dz (F. Dahmoune). oxygen in high quantities, can favor oxidative reactions. In the upper intestinal compartment, oxygen remains present and the lipid emulsification or micellization induced by the biliary salts could favor the pro-oxidant activity of iron ions ingested concomitantly with polyunsaturated fatty acid (PUFA)-carrying lipids (Kenmogne-Domguia, Moisan, Viau, Genot, \& Meynier, 2014). So, lipid oxidation of fatty acids could take place in the digestive tract before their intestinal absorption. Indeed, it is important to take into account that lipid oxidation products could be absorbed by intestinal cells (Kenmogne-Domguia et al., 2014) which could lead to an increase in oxidative stress in the plasma after their absorption. Thus, the inhibition of lipid peroxidation during ingestion is an important role of radical-scavenging dietary antioxidants. In the few studies investigating lipid oxidation in simulated gastric conditions of oil-in-water model emulsions, an effect of quercetin and $\alpha$-tocopherol on inhibition of heme-induced peroxidation was observed (Goupy, Vulcain, Caris-Veyrat, \& Dangles, 2007; 
Lorrain, Dangles, Genot, \& Dufour, 2009). A recent study (Kenmogne-Domguia et al., 2014) showed also that during in vitro digestion, emulsified lipids were oxidized, and endogenous tocopherols were consumed with a formation of a high amount of malonaldehyde at the end of the digestion. Many medicinal plants contain large amounts of antioxidants such as polyphenols, which possess significant antioxidant capacities associated with lower occurrence and lower mortality rates of several human diseases (Aidi Wannes et al., 2010). For this, contemporary dietary programs often recommend certain aromatic and medicinal plants as functional foods. The enrichment of lipid food by natural antioxidant was recently developed as a new way of phytochemical potential application. The enrichment of extra virgin olive oil (EVOO) by green tea phenolic compounds improved significantly the antiatherogenic properties of olive oil resulting in significant attenuation of atherosclerosis development (Rosenblat, Volkova, Coleman, Almagor, \& Aviram, 2008). Moreover, in a mixture of phenolic compounds, synergistic or antagonistic effects may occur and this could modulate the total antioxidant activity of the whole food. Subsequently, it is very important to take into account all these interactions when designing a functional food (PalafoxCarlos et al., 2012). According to our knowledge, there is none work about the effect of plant-enriched extra virgin olive oil on peroxidation of a phospholipid aqueous dispersion model under simulated gastrointestinal conditions.

The aim of this work is to evaluate for the first time the free radical scavenging effect and/or chelating ability of extra virgin olive oil enriched by myrtle extracts (obtained by both methods: microwave assisted extraction MAE and conventional extraction $\mathrm{CE}$ ) on egg yolk phosphatidylcholine/bile salts (EYPC/BS) aqueous dispersion oxidation under simulated intestinal conditions ( $\mathrm{pH} 7.4)$. The oxidation model was used to simulate the intestinal conditions $(\mathrm{pH}$ 7.4) of lipid peroxidation may occurring in small intestine during lipid digestion. For this, we have used EYPC as lipid substrate and bile salts as physiological detergent substances to stabilize the phospholipid aqueous dispersion formed by sonication. AAPH or $\mathrm{Fe}^{+3} /$ ascorbic acid system were used to initialize the phospholipid peroxidation. The cryo electronic microscopy was applied to visualize structure and size of formed lipid in the system used (data not shown).

\section{Materials and methods}

\subsection{Chemicals and standards}

All chemicals used were of analytical grade. Egg yolkphosphatidylcholine (EYPC), butylatedhydroxytoluene (BHT), and $2 \mathrm{~N}$ Folin-Ciocalteu reagent were purchased from Sigma Aldrich Chemical Co. (Saint Quentin Fallavier, France). Gallic acid and $2^{\prime}, 7^{\prime}$-dich loro-fluorescein were obtained from Merck (Darmstadt, Germany). Methanol used for chromatography was HPLC-grade supplied by Merck. Ethanol (EtOH) used for preparing standard solutions was from Prolabo (Paris, France). Chloroform was from Prolabo. 2.2'-A zobis-2-amidinopropane hydrochloride (AAPH) was from Biovalley (Conches, France). Ascorbic acid was purchased from Merck, France. Iron (III) chloride $\left(\mathrm{FeCl}_{3}, 6 \mathrm{H}_{2} \mathrm{O}\right)$ from Labosi-Fischer Scientific, France. Myricitrin was purchased from Roth Sochiel EURL (Lauterbourg, France).

\subsection{Plant material preparation}

M. communis leaves were collected in the region of Bejaia (Algeria), and dried in an oven at $40{ }^{\circ} \mathrm{C}$ until constant weight, then crushed and sieved to have a size less than $125 \mu \mathrm{m}$. The samples were stored in the dark at room temperature.

\subsection{Extraction procedure}

\subsubsection{Microwave assisted extraction (MAE) method}

The extraction procedure was previously described in our previous study (Dairi et al., 2014). Briefly, a domestic microwave oven (NN-S674MF, LG, Japan, $32 \mathrm{~L}, 1000 \mathrm{~W}$; variable in $100 \mathrm{~W}$ increments, $2.45 \mathrm{GHz}$ ) was used to extract myrtle leaf phenolic compounds. The leaf suspension ( $1 \mathrm{~g}$ of leaf powder per $20 \mathrm{~mL}$ of EtOH/water, $50 / 50 ; \mathrm{v} / \mathrm{v}$ ) was irradiated by microwaves $(700 \mathrm{~W}$ of power) for $1 \mathrm{~min}$. After MAE treatment, the sample was filtered and two other additional extractions were carried out. The final extract was stored at $4{ }^{\circ} \mathrm{C}$ until further use.

\subsubsection{Conventional extraction (CE) method}

Samples were extracted with EtOH/water (50/50; v/v) (Dairi et al., 2014). Briefly, $1 \mathrm{~g}$ of myrtle powder was mixed with $20 \mathrm{~mL}$ of hydroalcohol solvent and blended for $60 \mathrm{~min}$ with magnetic agitation at room temperature. After that, the process was the same as for MAE procedure.

\subsection{Identification and quantification of McPCs by RP-HPLC}

The chromatographic studies were performed as described in our previous report (Dairi et al., 2015) using an Agilent Model 1100 LC instrument (Agilent Technologies, Palo Alto, CA, USA) equipped with UV-Vis diode-array detector and rapid scan fluorescence spectrophotometer detector. The analytical data were evaluated using Chemstation software package. The analytical column employed was a 120 ODS $5 \mu \mathrm{m}, 150 \times 4.6 \mathrm{~mm}$ (Teknokroma, Barcelona, Spain). The column temperature was set at $25^{\circ} \mathrm{C}$. The mobile phase consisted of water with $0.5 \%$ acetic acid and $0.1 \%$ acetonitrile (A) and acetonitrile with $0.5 \%$ acetic acid (B). The gradient program was as follows: $0-20 \mathrm{~min}, 10 \% \mathrm{~B} ; 20-30 \mathrm{~min}, 45 \% \mathrm{~B} ; 30-$ $45 \mathrm{~min}, 45 \%$ B; $45-50 \mathrm{~min}, 10 \%$ B; $50-65 \mathrm{~min}, 10 \%$ B. The flow rate was set constant at $0.5 \mathrm{~mL} \mathrm{~min}^{-1}$ and the injection volume was $10 \mu \mathrm{L}$. The identification of each compound was based on a combination of retention time and spectral matching.

\subsection{Measurement of antioxidant activities}

\subsubsection{1,1-Diphenyl-2-picrylhydrazyl radical (DPPH') scavenging assay}

The antioxidant activity of sample extracts was measured by bleaching of the purple-colored solution of DPPH' under reduction by an antioxidant compound. The DPPH solution $\left(60 \mu \mathrm{mol} \mathrm{L}^{-1}\right)$ was prepared in methanol, and $3 \mathrm{~mL}$ of this solution was mixed with $100 \mu \mathrm{L}$ of samples extracts or of standard BHA or $\alpha$-tocopherol at various concentrations. The samples were incubated for $20 \mathrm{~min}$ at $37^{\circ} \mathrm{C}$ in a water bath, and then the absorbance decrease was measured at $515 \mathrm{~nm}$ (Dudonne, Vitrac, Coutiere, Woillez, \& Merillon, 2009).

The percentage of loss of DPPH absorbance was calculated according to the following equation: \% loss of $\mathrm{DPPH}^{-}=[(\mathrm{AC}$ (DPPH.) - AA (sample)/AC (DPPH.) $] \times 100$, where AC $\left(\mathrm{DPPH}^{\circ}\right)$ is the control absorbance at time $=0 \mathrm{~min}$; and $\mathrm{AA}$ (sample) is the absorbance of $\mathrm{DPPH}^{*}$ in the presence of antioxidant at time $=20$ min. The antioxidant activity of each test sample and standard is expressed in terms of concentration required to reduce DPPH. absorbance by half $\left(\mathrm{IC}_{50}\right.$ expressed as $\mathrm{mg} \mathrm{L}^{-1}$ ) and calculated from the log-dose inhibition curve.

\subsubsection{Oxygen radical absorbance capacity (ORAC)}

ORAC values were measured with fluorescence spectrometer (Victor ${ }^{2}$ Wallac-Perkin-Elmer) by inhibition of 2', 7'dichlorofluorescein (DCF) consumption (Ishimoto et al., 2011) with slight modifications. Briefly, all samples and reagents were dissolved in $10 \mathrm{mmol}$ phosphate $\mathrm{L}^{-1} / 150 \mathrm{mmol} \mathrm{NaCl} \mathrm{L}-1$ buffers (PBS) at $\mathrm{pH}$ 7.4. $50 \mu \mathrm{L}$ 
test samples or $50 \mu \mathrm{L}$ Trolox solutions $\left(0-20 \mu \mathrm{mol} \mathrm{L}^{-1}\right), 100 \mu \mathrm{L} \mathrm{DCF}$ solution $\left(50 \mathrm{nmol} \mathrm{L}^{-1}\right)$, and $100 \mu \mathrm{L}$ AAPH solution $\left(20 \mathrm{mmol} \mathrm{L}^{-1}\right)$ were added to the wells of a 96-well plate. The fluorescence was recorded every $1 \mathrm{~min}$ for $90 \mathrm{~min}$ at $485 \mathrm{~nm}$-excitation and $535 \mathrm{~nm}$-emission wavelengths. A calibration curve was established by plotting the period of time needed to obtain 50\%-fluorescence decay versus the Trolox concentrations. ORAC levels are expressed as mole of Trolox equivalent (TE) per mole of antioxidant (pure compounds) or per mole of GAE for myrtle extracts.

2.5.3. Aqueous dispersion preparation with egg yolk phosphatidylcholine and bile salts and evaluation of sample extracts effects on AAPH or $\mathrm{Fe}^{+3} /$ ascorbic acid-mediated oxidation

The phospholipid aqueous dispersion was prepared as described previously by Dairi et al. (2014). Briefly, egg yolk phosphatidylcholine (EYPC) in chloroform was flushed with nitrogen gas to remove the solvent, and then bile salts, as emulsifying agent, and $10 \mathrm{~mL}$ of phosphate buffered saline (PBS, pH 6.5) were added. The mixture was sonified for $5 \times 30 \mathrm{~s}$, and incubated at $37^{\circ} \mathrm{C}$ during $30 \mathrm{~min}$, and filtered to $0.2 \mu \mathrm{m}$. Finally, the $\mathrm{pH}$ was adjusted to 7.4 .

Aqueous dispersion oxidation was evaluated by monitoring the production of conjugated dienes until $150 \mathrm{~min}$. Briefly, lipid dispersion sample $(1 \mathrm{~mL})$ was oxidized at $37^{\circ} \mathrm{C}$ with AAPH $(0.5 \mathrm{~mL})$ or $\mathrm{Fe}^{+3} /$ Ascorbic acid to achieve final concentrations of $5 \mathrm{mmol} \mathrm{L}^{-1}$ or $50 / 50 \mu \mathrm{mol} \mathrm{L}^{-1}$ respectively, and PBS $(0.5 \mathrm{~mL})$ containing different tested concentrations of samples or pure standards was added. Results were evaluated as the ratio of tested antioxidants AUC to that of control, and are expressed as percentage (Dairi et al., 2014). The protective effect (PE) was calculated using the following formula: protective effect $=100 \%-\mathrm{AUC} \%$ of the sample tested. An increased stability ratio was calculated using the following equation: stability ratio $(\%)=\left[\left(\mathrm{PE}^{+}-\mathrm{PE}^{-}\right) / \mathrm{PE}^{-}\right] \times 100$, with ${ }^{+}$and ${ }^{-}$denoting enriched or un-enriched EVOO with antioxidants.

\subsection{Determination of vitamin $E$ content during oxidation of $E Y P C / B S$} aqueous dispersion

After the preparation of lipid extract from aqueous dispersion as previously reported (Dairi et al., 2014), vitamin E ( $\boldsymbol{\alpha}$-tocopherol and $\gamma$-tocopherol) analysis was carried out using simple isocratic elution that consisted of mobile phase water/methanol mixture $(3 / 97, \mathrm{v} / \mathrm{v})$ with a flow rate of $0.8 \mathrm{~mL} \mathrm{~min}^{-1}$ for $12 \mathrm{~min}$. $\delta$ tocopherol was used as an internal standard. HPLC separation was performed on a Lichrocart 125-4 (5 $\mu$ m-particle size) column (Merck, France). The detector was set at $292 \mathrm{~nm}$.

\subsection{Preparation of extra virgin olive oil enrichment}

Weighed quantities of McPC extracts were dissolved in an appropriate volume of $\mathrm{EtOH} /$ water $(50 / 50 ; \mathrm{v} / \mathrm{v})$ to obtain the desired final mass concentration (200 $\mathrm{mg} \mathrm{GAE} \mathrm{kg}^{-1}$ oil). Then, $500 \mu \mathrm{L}$ of this solution was added to extra virgin olive oil ( $500 \mathrm{~g}$ ) stepwise under vigorous stirring for $30 \mathrm{~min}$ (Bouaziz, Fki, Jemai, Ayadi, \& Sayadi, 2008). Then, the mixture was sonified for $30 \mathrm{~s}$ to allow the complete dissolution of the extract. The same procedure was also done for BHT and $\alpha$-tocopherol as standards to achieve a final mass concentration of $200 \mathrm{mg} \mathrm{kg}^{-1}$ oil. Samples were stored in the dark at $6{ }^{\circ} \mathrm{C}$ until use.

\subsection{Extraction and quantification of phenolic compounds from extra virgin olive oil}

Samples (2.5 g of oil enriched with McPC or $\alpha$-tocopherol or BHT and unenriched oil) were dissolved in $5 \mathrm{~mL} n$-hexane and extracted with $5 \mathrm{~mL}$ of a methanol/water mixture $(60 / 40, \mathrm{v} / \mathrm{v})$. The resulting mixture was shaken vigorously by means of a mechanical shaker (Vortex) for $2 \mathrm{~min}$ and centrifuged at $3000 \mathrm{~g}$ for $10 \mathrm{~min}$. The polar fraction (methanolic phase) was recovered, whereas the non-polar phase (hexane) followed other methanolic extractions. The fractions obtained were combined and stored at $4{ }^{\circ} \mathrm{C}$ (Kalantzakis, Blekas, Pegklidou, \& Boskou, 2006). Then, total phenolic compounds concentration was estimated by FolinCiocalteu's assay, with absorbance monitored at $760 \mathrm{~nm}$ (Monde et al., 2011). The result is expressed on a dry weight basis as $\mathrm{mg}$ of gallic acid equivalents (GAE) per $\mathrm{kg}$ of olive oil (mg GAE kg${ }^{-1}$ ).

\subsection{Statistical analysis}

Analyses were carried out three times or more and results are reported as mean values \pm standard deviation (SD). Data were compared on the basis of the mean values. Differences among means of variety groups were tested using a Tukey-Kramer HSD (SoftwareJMP version 7.0) with a significance level of 0.05 .

\section{Results and discussion}

\subsection{Phenolic compound identification and quantification by RP-HPLC- DAD-FLD}

The chromatogram profiles of Myrtus communis extract obtained with both used methods (MAE and CE) acquired at 280 and $350 \mathrm{~nm}$ and at $230 / 350 \mathrm{~nm}$ (excitation/emission wavelengths) showed close composition of phenolic compounds (Table 1 and Supplementary data). These results confirm that microwave extraction can be an alternative to the conventional method allowing a good recovery of phenolic compounds which is consistent with several previous studies (Proestos \& Komaitis, 2008). At $280 \mathrm{~nm}$, the chromatogram reported the presence of an important peak after $4.1 \mathrm{~min}$ and it was quantified as mg gallic acid equivalents per $g$ dry extract ( $\mathrm{mg} \mathrm{GAE} \mathrm{gE}^{-1}$ ): due to the lack of standards it can only supposed to be galloyl quinic acid $(273 \mathrm{~nm})$,

Table 1

Identification and quantification by RP-HPLC-DAD-FLD of phenolic compounds in Myrtus communis leaves.

\begin{tabular}{|c|c|c|c|c|c|}
\hline Phenolic compound & $\operatorname{Tr}(\min )$ & $\chi_{\mathrm{UV}-\mathrm{vis}} \max (\mathrm{nm})$ & Equation; $\mathrm{R}^{2}$ & MAE extract & CE extract \\
\hline Galloylquinic acid" & 4.08 & 273 & - & $7.33 \pm 0.06$ & $7.66 \pm 0.01$ \\
\hline Gallic acid & 4.82 & 275 & $\begin{array}{l}y=52.834 x-23.727 \\
R^{2}=0.998\end{array}$ & $3.53 \pm 0.15$ & $3.31 \pm 0.03$ \\
\hline Myricetin $3-O$ galactoside & 30.63 & 260,360 & - & $2.38 \pm 0.18$ & $2.37 \pm 0.04$ \\
\hline Myricetin 3-O rhamnoside & 31.54 & 265,360 & $\begin{array}{l}y=28.737 x-5.614 \\
R^{2}=0.999\end{array}$ & $12.26 \pm 0.84$ & $11.78 \pm 0.06$ \\
\hline Ellagic acid" & 31.93 & 255,368 & - & $0.84 \pm 0.03$ & $0.88 \pm 0.02$ \\
\hline
\end{tabular}

Gallic acid and myricitrin are expressed as mg per $\mathrm{g}$ of dry extract and identified according to their pure standard data.

The result is the mean of three experiments $(n=3) \pm$ standard deviation (SD).

"The compounds were identified according to literature data and the result is expressed as mg gallic acid equivalent per g of extract for galloyl quinic acid and as mg myricetin 3-0- rhamnoside equivalent per g of extract for myricetin 3-O-galactoside and ellagic acid. 
according to spectral and previously reported data (Annalisa Romani, Campo, \& Pinelli, 2012). Gallic acid was identified by comparing its retention time and spectral data with its authentic standard using HPLC-DAD signal. In previously reported works, the phenolic compound composition of Italian (A Romani, Pinelli, Mulinacci, Vincieri, \& Tattini, 1999) and Tunisian myrtle leaves (Aidi Wannes et al., 2010; Messaoud, Laabidi, \& Boussaid, 2012) was examined and it was found the presence of different compounds belonging to the phenolic acid family, with gallic acid in Tunisian variety being more important than in the Italian variety. At $350 \mathrm{~nm}$, we have also identified and quantified derivatives of myricetin in myrtle leaf extracts. The main important flavonoid detected was myricitrin (myricetin-3-0 rhamnoside), identified by its retention time, spectral and fluorescence data in comparison with the standard. It was 3.47 and 3.55 times more abundant than gallic acid in myrtle extracts (MAE and CE respectively) which is consistent with the previously reported Italian myrtle leaf composition (Romani et al., 2012; Romani et al., 1999), whereas literature data report that gallic acid is more important than myricetin 3-Orhamnoside in Tunisian myrtle leaves (Aidi Wannes et al., 2010; Messaoud et al., 2012). These differences supposed a variety effect on the phenolic compound composition of myrtle leaves. The other myricetin derivative, myricetin-3-O-galactoside, and ellagic acid, were identified according to the finding of Romani et al. (2012).

\subsection{Antioxidant activities of Myrtus communis leaf polyphenolic compounds}

\subsubsection{Oxidation induced by AAPH or $\mathrm{Fe}^{+3} /$ ascorbic acid system}

3.2.1.1. AAPH-induced oxidation in a model of phospholipid/BS aqueous dispersion. The AAPH-induced phospholipid oxidation of aqueous dispersion stabilized by bile salts at the $\mathrm{pH} 7.4$ (final intestinal conditions) was investigated. Results were expressed as AUC percentage. Lower AUC value indicates higher antioxidant activity. The lipid structures in our model were formed by egg yolk phosphatidylcholine (EYPC) and bile salt (BS) with a ratio (EYPC/BS) of 0.8 and were characterized by cryo-Transmission Electron Microscopy (cryo-TEM) which revealed the presence of small unilamellar vesicles $(100 \mathrm{~nm}$ average size) and small micelles (15 nm average size) (data not shown). Fig. 1a shows that when the concentration of McPCs increased, the production of conjugated dienes $(C D)$ decreased significantly in a dose dependant manner. The ability of $M$. communis leaf extracts to scavenge free radicals could be attributed to an overall participation of their active constituents, such as phenolic acids and flavonoids. As described above, myrtle extract analysis revealed the presence of gallic acid and myricetin derivatives (as myricitrin) but it was reported also the presence of other compounds (Romani et al., 2012) which may also contribute to the antioxidant activity of the myrtle extract. Lipid oxidation in dispersed lipid phase is prevalent at the oil-water interface, where lipid hydro-peroxides are decomposed into free radicals by transition metals or other oxidant agents. Thus, free radical scavenging antioxidants are believed to be most effective in lipid dispersions when they accumulate at the oil-water interface (Losada Barreiro, Bravo-Díaz, Paiva-Martins, \& Romsted, 2013; Yuji et al., 2007). In our study, the lipid peroxidation was induced by free radicals derived from AAPH decomposition which occurred in the aqueous phase. Gallic acid is a water soluble antioxidant and is predominantly located in the aqueous-interfacial regions of the phospholipid dispersion (Losada Barreiro et al., 2013) where it may interact with AAPH derived radicals and consequently reduce the production of conjugated dienes. In addition, myricitrin, one of the main flavonol of myrtle extract, had a strong scavenging effect as depicted in our former work (Dairi et al., 2014). In ORAC assay, myricitrin had a better ability to scavenge free radicals from AAPH decomposition
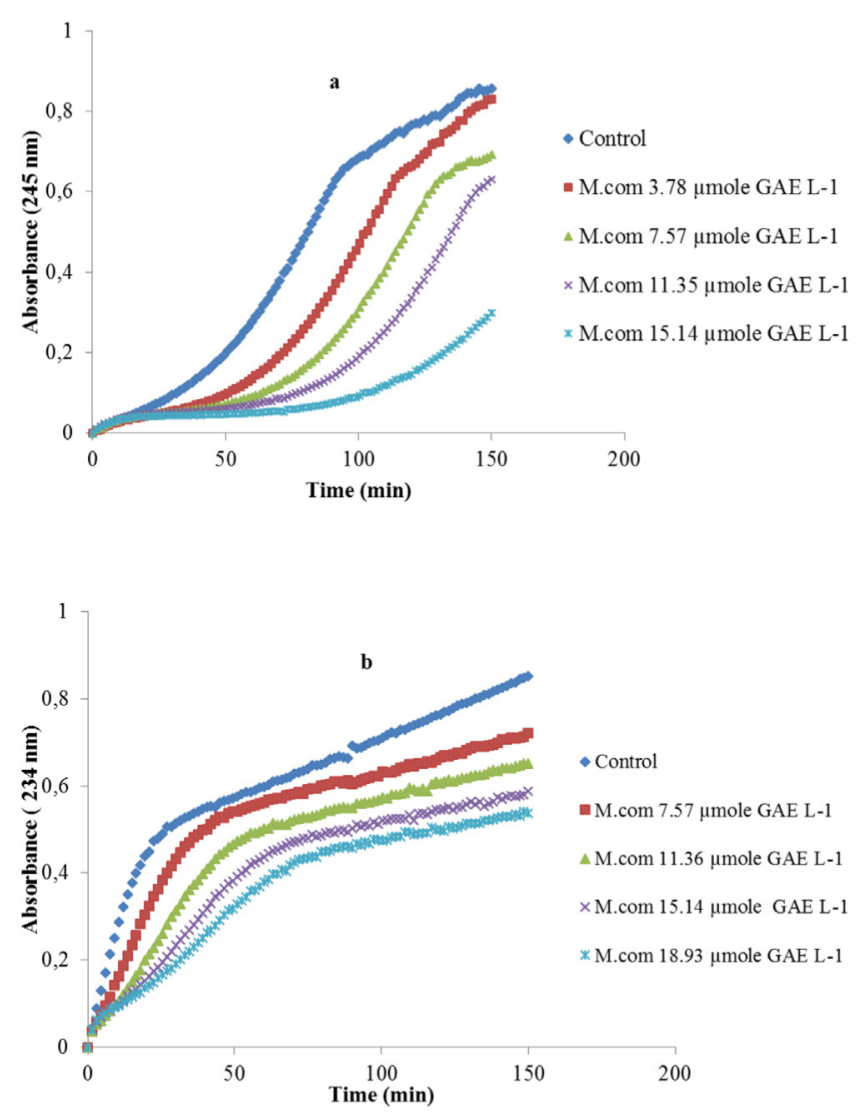

Fig. 1. Kinetic oxidation of phospholipid aqueous dispersion induced by AAPH derived radicals (a) or $\mathrm{Fe}^{+3} / \mathrm{AsA}$ system (b) at $\mathrm{pH} 7.4$ in the presence or absence (control) of M. communis polyphenolic compounds (MAE method). Kinetic of CD production with McPC (CE method) gives similar curves.

than myrtle extract, and thus, may contribute to the total protective effect of this extract. On the other hand, myricitrin, less polar antioxidant than gallic acid, can be near to the aqueous dispersion interface where the peroxidation by AAPH derived radicals is predominant and two major mechanism of action may occur: neutralization of organic radicals formed by thermal decomposition of AAPH occurred in aqueous phase and possibly scavenging of lipid alkoxyl and peroxyl radicals by acting as hydrogen donors and/or chain breaking antioxidants.

3.2.1.2. Phospholipid oxidation induced by Iron/Ascorbic acid system. To investigate the ability of myrtle extract to chelate divalent ferrous ions $\left(\mathrm{Fe}^{+2}\right)$, we studied phospholipid oxidation mediated by $\mathrm{Fe}^{+3} / \mathrm{AsA}$ system. This system is currently used to initialize the peroxidation (Bondet, Cuvelier, \& Berset, 2000). In such a system, $\mathrm{Fe}^{+2}$ ions produced by AsA reduction of $\mathrm{Fe}^{+3}$ react with traces of hydro-peroxides to give primary radicals producing the lipoperoxidation (Bondet et al., 2000). Fig. 1b shows the kinetic of conjugated diene production observed at $234 \mathrm{~nm}$ during the $\mathrm{Fe}^{3+} /$ AsA system-induced oxidation of the EYPC/BS aqueous dispersion model. This oxidation kinetic was biphasic; we observed firstly an important increase in oxidation rate (without apparition of a lag phase, unlike that observed in the AAPH assay: see Fig. 1a) and then a linear phase running for more than $2 \mathrm{~h}$ without reaching a plateau. The addition of myrtle extracts to the EYPC/BS aqueous dispersion extended the initiation phase and decreased the peroxidation rate with a dose-depend effect. A recent work of Gonçalves, Gomes, Costa, and Romano (2013) showed that the amount of $\mathrm{Fe}^{2+}$-induced lipid peroxidation in mouse brain homogenates decreased when aqueous myrtle extract was used. These 
observations could relate the protection against lipid peroxidation to the ability of $M$. communis extracts to scavenge the free radicals formed by $\mathrm{Fe}^{2+}$ degradation of preformed hydro-peroxides (Oboh, Puntel, \& Rocha, 2007) and /or to chelate $\mathrm{Fe}^{2+}$ (Mladěnka et al., 2011; Oboh et al., 2007).

To investigate the oxidant type effect on oxidative damage of lipid bilayers in absence or presence of antioxidants, myrtle extracts, myricitrin and $\alpha$-tocopherol were tested at equivalent concentration of $10 \mu \mathrm{mol} \mathrm{GAE} \mathrm{L^{-1 }}$ (for McPC complex mixtures) and $10 \mu \mathrm{mol} \mathrm{L}^{-1}$ (for pure compounds) on AAPH or $\mathrm{Fe}^{+3} / \mathrm{AsA}$ system-induced phospholipid oxidation. As shown in the Fig. 2, AUC values of the EYPC/BS aqueous dispersions containing myrtle extracts or myricitrin were lower in AAPH than in $\mathrm{Fe}^{+3} / \mathrm{AsA}$ system. Myrtle (MAE and CE) extracts increased the phospholipid stability by factors of 1.43 and 1.46 respectively in AAPH compared to $\mathrm{Fe}^{+3} / \mathrm{AsA}$ system. If myricitrin increased strongly the EYPC/BS aqueous dispersion stability in AAPH system, when metal ions were added as oxidant agent, a drastic loss (c.a. 50\%) of stability was observed. For $\alpha$-tocopherol, an opposite effect was observed compared to myricitrin. The EYPC/BS aqueous dispersion stability increased strongly (c.a. $37.0 \%$ of stability gain) in $\mathrm{Fe}^{+3} / \mathrm{AsA}$ system compared to AAPH (Gutiérrez et al., 2003). Under our conditions, $\alpha$-tocopherol had the most effective protective effect. On the other hand, when metals ions (cupric and ferric ions) were added as inducer of peroxidation, the loss of stability for liposomes containing flavonols and specially myricetin was previously observed (Gordon \& Roedig-Penman, 1998) which was consistent with our finding. This could be interpreted by the fact that at $\mathrm{pH} 7.4$, biles salt molecules present in the phospholipid vesicle surface were nearly completely negatively charged $(\mathrm{pKa}<\mathrm{pH})$ which increased strongly the attraction of $\mathrm{Fe}^{2+} / \mathrm{Fe}^{3+}$ ions present in the aqueous phase. Moreover, the decomposition of flavonols (as myricitrin) catalyzed by these ions may occurred (Gordon \& Roedig-Penman, 1998). This interaction between $\mathrm{Fe}^{2+}$ ions and flavonols (myricitrin) is consistent with the flavonol location near to the surface of bilayers, since metal ions are unlikely to penetrate into the vesicle hydrocarbon region (Gordon \& Roedig-Penman, 1998). Moreover, Vitamin E (as $\alpha$-tocopherol) acts as a chain-breaking antioxidant that quickly reduces $1-2$ equivalents of lipid peroxyl radicals (inhibition of propagation) (Goupy et al., 2007). The two oxidants used, $\mathrm{AAPH}$ and $\mathrm{Fe}^{3+} / \mathrm{AsA}$, were located in the aqueous

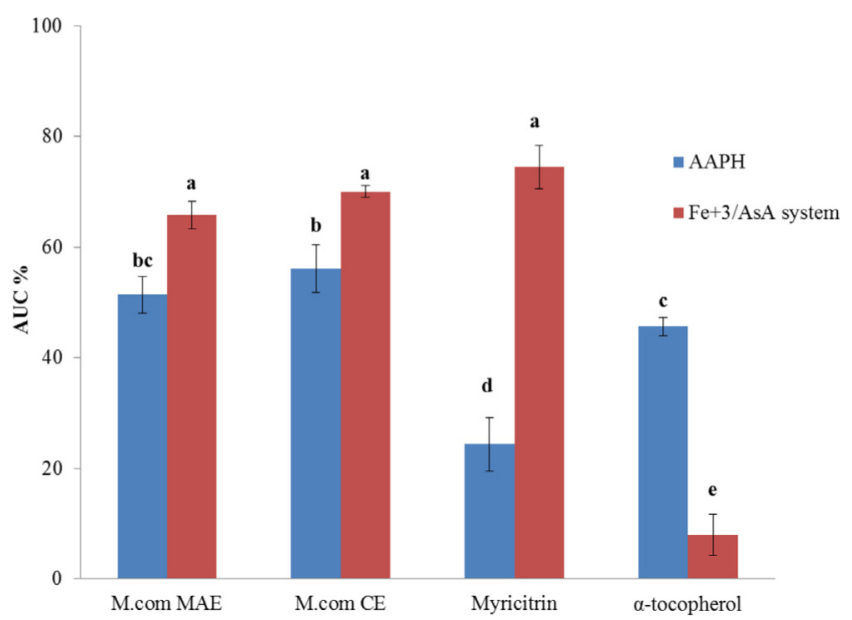

Fig. 2. Comparison of antioxidant activity of myrtle extracts, myricitrin and $\alpha$ tocopherol in inhibiting phospholipid aqueous peroxidation induced by different free radicals species. The final concentration of antioxidant used was $10 \mu \mathrm{mol} \mathrm{GAE} \mathrm{L}^{-1}$ (myrtle extracts) or $10 \mu \mathrm{mol} \mathrm{L}^{-1}$ for myricitrin and $\alpha$-tocopherol. Results are expressed as means of three experiments \pm SD. Means with different letters for each compound are significantly different at $p<0.05$. phase and at/or near the phospholipid bilayer surface as myricitrin, when $\alpha$-tocopherol was inside. This localization difference may explain the difference between $\alpha$-tocopherol and myricitrin action in these two systems. This result might be supported by the work of Gutiérrez et al. (2003) who showed that when both antioxidant and free radical coincide in the same place, the antioxidant effect is amplified. These data concluded that there is an antioxidantstructure relationship for peroxidation inhibition and that the "polar paradox" was not confirmed for all the study conditions used.

\subsection{Could M. communis PCs improve anti-oxidative activity of extra virgin olive oil?}

\subsubsection{Total phenolic compound content estimation of enriched extra virgin olive oil}

Total phenolic compound of McPC extracts was determined by Folin-Ciocalteu's assay and revealed that both myrtle MAE and CE extracts gave very close levels of phenolic compounds $\left(176.4 \pm 6.8\right.$ and $181.0 \pm 6.3 \mathrm{mg} \mathrm{GAE} \mathrm{g}^{-1} \mathrm{DW}$, respectively) (Data no shown). In this study, phenolic compound content of EVOO control evaluated by Folin-Ciocalteu's assay was $248.6 \pm 3.4 \mathrm{mg} \mathrm{GAE} \mathrm{kg}^{-1}$ of oil, which is in accordance with the literature data. The enrichment of EVOO increased its phenolic compound content by $43.4 \%$ and $38.4 \%$ corresponding to $355.9 \pm 1.6 \mathrm{mg}$ and $344.1 \pm 2.7 \mathrm{mg} \mathrm{GAE} \mathrm{kg}^{-1}$ for MAE and CE-enriched EVOO (EEVOO) respectively.

\subsubsection{Antioxidant capacity estimation of McPC-enriched extra virgin olive oil}

3.3.2.1. 1,1-Diphenyl-2-picrylhydrazyl radical (DPPH.) assay. In this present work, the scavenging capacity of DPPH radicals by EEVOO extracts was evaluated (Table 2) and the results are expressed as loss percentage of DPPH radicals. EVOO control extract (a concentration of $23.6 \mu \mathrm{mol} \mathrm{GAE} \mathrm{L}^{-1}$ ) reduced the DPPH radical by a \% loss of $44.3 \pm 1.7 \%$. The EVOO supplementation with different antioxidant compounds increased significantly the loss of DPPH radical by factors of $107.8,114.4$ and $9.9 \%$ for myrtle MAE or CE extracts or BHT, respectively. Moreover, when $\alpha$-tocopherol was used, no additional loss effect against DPPH radical was observed. Several authors have determined the antiradical and antioxidant activities of hydrophilic fraction of EVOO by measuring the consumption of the DPPH radical (Minioti \& Georgiou, 2010). The antioxidant activity-phenolic compound content relationship was widely studied by several works, showing clearly that an increase in phenolic compound content correlated with a higher scavenging effect of the extract (Messaoud et al., 2012). This could explain the highest scavenging activity of DPPH radicals by McPC-EEVOO extracts. Moreover, the enrichment of edible oil (as olive oil) by olive leaf extract increased significantly the scavenging effect against DPPH radical (Chiou, Kalogeropoulos, Salta, Efstathiou, \& Andrikopoulos, 2009), and this observation may also support our finding. On the other hand, the qualitative composition of the

Table 2

Evaluation of antioxidant activity of extra virgin olive oil (EVOO) enriched by myrtle (MAE and CE) extracts, BHT or $\alpha$-tocopherol using DPPH and ORAC assay.

\begin{tabular}{lll}
\hline EVOO type & DPPH $($ loss $\%)$ & ORAC $\left(\right.$ TE mol GAE mol $\left.{ }^{-1}\right)$ \\
\hline EVOO control & $44.3 \pm 1.7^{\mathrm{b}}$ & $5.3 \pm 0.0^{\mathrm{b}}$ \\
EVOO + M.com MAE & $92.1 \pm 0.7^{\mathrm{a}}$ & $7.4 \pm 0.2^{\mathrm{a}}$ \\
EVOO + M.com CE & $95.0 \pm 0.5^{\mathrm{a}}$ & $7.3 \pm 0.5^{\mathrm{a}}$ \\
EVOO + BHT & $48.7 \pm 2.7^{\mathrm{b}}$ & $7.5 \pm 0.4^{\mathrm{a}}$ \\
EVOO + $\alpha$-tocopherol & $44.0 \pm 3.8^{\mathrm{b}}$ & $5.7 \pm 0.0^{\mathrm{b}}$ \\
\hline
\end{tabular}

Assays are performed in triplicate $(n=3)$ and data are expressed as mean \pm standard deviation. Means followed by different letters in the same column are significantly different $(p<0.05)$. The same volume of each EVOO type was tested in DPPH and ORAC assay. 
studied olive oils was analyzed in our previous work (Dairi et al., 2014) using reversed phase dispersive liquid-liquid microextraction (RP-DLLME)-HPLC-DAD-FLD and it was revealed that the enriched oil presented a new composition with the presence of galloyl quinic acid, gallic acid and myricitrin, and it was shown that these compounds contributed to the increase of olive oil oxidative stability by decreasing the production of oxidation primary product. According to all these observations, it may be suggested a possible synergic or cooperative effect between myrtle and EVOO phenolic compounds against DPPH radical loss by their scavenging ability.

3.3.2.2. The Oxygen radical absorbance capacity (ORAC) method. The ORAC assay was applied also to assess the antioxidant activity of EEVOO and is expressed as mol TE mol GAE ${ }^{1}$ for EVOO extracts and as mol TE mol-1 of pure antioxidants. As can be seen in Table 2 , total antioxidant capacity value of EVOO extract was $5.35 \pm 0.06 \mathrm{~mol} \mathrm{TE} \mathrm{mol} \mathrm{GAE}{ }^{-1}$. The EVOO supplementation increased significantly the protection of fluorescein consumption during ORAC assay by a factor of $27.7 \pm 3.7,26.4 \pm 5.4$ and $28.2 \pm 3.1 \%$ for MAE or CE extract-and BHT-enriched EVOO, respectively. Moreover, when $\alpha$-tocopherol was used, the protective effect on fluorescein consumption was only increased by a factor of $7.0 \pm 1.4 \%$, so showing the lowest ORAC value. ORAC values observed were lower than the DPPH loss values. This finding was consistent with our previous study showing that myrtle extract had a higher scavenging effect under DPPH assay than when ORAC assay was applied (data not shown). Thus, the antioxidant activity value depends of the evaluation method used and the reactivity of phenolic compounds towards different free radicals. According to the literature, incorporation of olive cake phenolic extract in virgin olive oil improved the antioxidant activity of enriched olive oil, evaluated by using the ORAC assay (Suárez, Romero, Ramo, \& Motilva, 2011).

3.3.2.3. AAPH or $\mathrm{Fe}^{+3} / \mathrm{AsA}$ system-induced peroxidation of $E Y P C / B S$ aqueous dispersion model. The ability of McPC-EEVOO to prevent the oxidation of EYPC/BS aqueous dispersion induced by different oxidants as $\mathrm{Fe}^{+3} / \mathrm{AsA}$ system or AAPH-derived radicals was investigated. The first reading of the result showed that McPCs could promote significantly the protective effect of EVOO against oxidation (Fig. 3). The EVOO control (without enrichment) showed a lower oxidation rate in AAPH system than in $\mathrm{Fe}^{+3} /$ AsA system, giving a protective effect of $77.2 \pm 1.1$ and $63.6 \pm 0.8 \%$, respectively. This supposes that EVOO phenolic compounds act efficiently against lipid peroxidation by scavenging AAPH-derived radicals more than chelating $\mathrm{Fe}^{+2}$ ions. It was previously shown that hydroxytyrosol (the main and the potent antioxidant in virgin olive oil) could serve as good scavenger of aqueous peroxyl radicals near the membrane surface and as powerful antioxidant against lipid peroxidation in phospholipid bilayers induced by aqueous oxygen radicals (Paiva-Martins \& Gordon, 2002; Saija et al., 1998). A latter work showed that hydroxytyrosol (with its catechol structure) was able to prevent lipid peroxidation of rat liver microsomes induced by Iron/ascorbate, when tyrosol possessing only one hydroxyl function was found to be inactive (Gutierrez, de la Puerta, \& Catalá, 2001). The feature determining the chelating activity of an antioxidant was clearly proved to be the presence of a catechol moiety (Mladěnka et al., 2011) and the lack of this structure for tyrosol and apigenin (identified in our EVOO sample) could contribute to explain the difference of protective effect found between the two oxidants used to initialize the phospholipid bilayer oxidation.

The supplementation of EVOO with phenolic compounds increased significantly the phospholipid stability in $\mathrm{Fe}^{+3} / \mathrm{AsA}$ system. The protective effect in decreasing order was $85.0 \pm 1.4$, $85.8 \pm 1.3,75.9 \pm 1.0$ and $70.5 \pm 0.9 \%$ for myrtle MAE and $\mathrm{CE}$

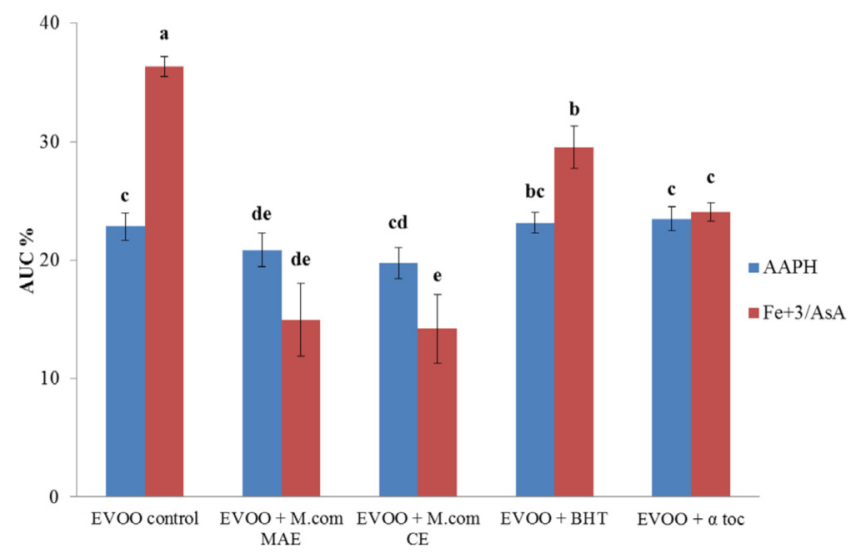

Fig. 3. Effect of myrtle extracts (MAE or CE methods), $\boldsymbol{\alpha}$-tocopherol or BHT enriched-EVOO phenolic compounds on the phospholipid peroxidation induced by AAPH or $\mathrm{Fe}^{+3} /$ AsA system. The same volume of each olive oil type was added to phospholipid aqueous dispersion oxidation. Means followed by different letters are significantly different $(p<0.05)$.

extracts, $\boldsymbol{\alpha}$-tocopherol and BHT respectively, corresponding to an increase in the phospholipid stability of $33.6,34.8,19.3$ and $10.7 \%$ for the above antioxidants respectively, compared to EVOO control. Our result showed clearly that myrtle extract was significantly more effective than $\alpha$-tocopherol and BHT $(p<0.05)$ in $\mathrm{Fe}^{+3} / \mathrm{AsA}$ system-induced phospholipid oxidation. This result was in contrast with that obtained when myrtle extracts and $\alpha$-tocopherol were used individually in the same oxidation conditions. There are synergistic and antagonistic interactions between flavonoids that may so explain results obtained when measuring the antioxidant effect of a whole food extract (Hidalgo, Sánchez-Moreno, \& de PascualTeresa, 2010). The new composition of EEVOO extract could improve the small unilamellar vesicle stability by synergistic or additive effects between various antioxidants present in the extract (from EVOO and McPCs) during oxidation mediated by $\mathrm{Fe}^{+3} / \mathrm{AsA}$ system. In addition, the work of Goupy et al. (2007) who studied the heme-induced peroxidation of linoleic acid reported that there was a synergic effect between quercetin and $\alpha$-tocopherol and supposed that this latter could regenerate some quercetin oxidation products still endowed with a reducing activity. The work of Marinova et al. (2008) showed also that myricetin aglycon had a best synergistic effect with $\alpha$-tocopherol and demonstrated that this lipo-soluble antioxidant can regenerate myricetin during autoxidation of triglyceride sunflower oil at $100{ }^{\circ} \mathrm{C}$. So, we were interested in the evaluation of EYPC vitamin E ( $\alpha$-and $\gamma$ tocopherol) content during $\mathrm{Fe}^{+3} / \mathrm{AsA}$ mediated phospholipid BS aqueous dispersion oxidation. Results presented in the Fig. 4 showed that after $150 \mathrm{~min}$ of phospholipid oxidation without any exogenous antioxidant, $\alpha$ - and $\gamma$-tocopherols were degraded at $79.7 \pm 0.9$ and $51.6 \pm 6.7 \%$ respectively, which means that these endogenous antioxidants could act as inhibitors of the lipid peroxidation. The addition of EVOO extracts decreased significantly $(\mathrm{p}<0.05)$ the vitamin E consumption to $28.6 \pm 6.9$ and $9.8 \pm 3.8 \%$ for $\alpha$ - and $\gamma$-tocopherols, respectively. This result corresponded to an increased protective effect by a factor of 2.8 and 5.3 for the above lipo-soluble antioxidants, respectively. This may be explained by the regeneration of vitamin E by EVOO phenolic compounds present in the extract, since olive oil phenolic compounds were located near to the phospholipid membrane (Paiva-Martins \& Gordon, 2002). All these results suggested that during $\mathrm{Fe}^{+3} / \mathrm{AsA}-$ induced phospholipid oxidation, EVOO phenolic compounds can serve as chelating agents of ferrous ions $\left(\mathrm{Fe}^{+2}\right)$ ions and/or as scavenging agents of free radicals produced by ferrous ions and/or as regenerating agent of $\alpha$-tocopherol. 


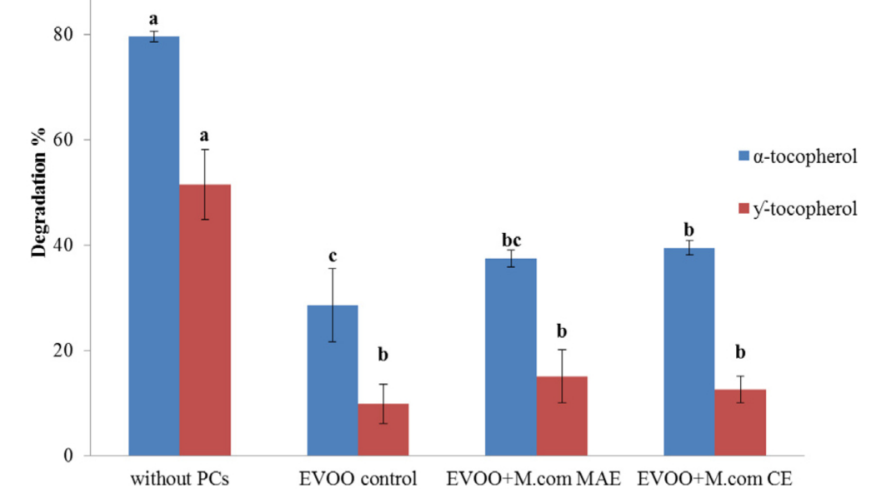

Fig. 4. Vitamin $\mathrm{E}$ ( $\alpha$ and $\gamma$-tocopherol) degradation during $\mathrm{Fe}^{+3} /$ ascorbic acid system-induced phospholipid oxidation in absence or presence of myrtle extractEEVOO extracts. The degradation rate was calculated as follow: Vitamin $E\left(t_{0 m i n}\right)-$ Vitamin $\mathrm{E}\left(\mathrm{t}_{150 \mathrm{~min}}\right) /$ vitamin $\mathrm{E}\left(\mathrm{t}_{0 \mathrm{~min}}\right) \times 100$. Means followed by different letters are significantly different $(p<0.05)$.

On the other hand, EEVOO extracts showed an increase of vitamin $\mathrm{E}$ depletion during phospholipid oxidation induced by $\mathrm{Fe}^{+3} /$ AsA compared to EVOO control extract. McPC-EEVOO extracts (MAE and CE) caused a vitamin E depletion of $37.4 \pm 1.6$ and $39.5 \pm 1.8 \%$ for $\alpha$-tocopherol, and $15.1 \pm 5.0$ and $12.5 \pm 2.5 \%$ for $\gamma$ tocopherol, respectively. These results were consistent with that concerning the contribution of vitamin $\mathrm{E}$ to regenerate some oxidized phenolic compounds (Goupy et al., 2007; Marinova et al., 2008). Thus, in addition to the possible action mechanisms of EVOO during $\mathrm{Fe}^{+3} / \mathrm{AsA}^{-i n d u c e d}$ phospholipid oxidation, McPCEEVOO could improve the total chelating activity and interact positively with bilayer membrane to scavenge lipid radicals. All these effects may be supported by the regeneration of some phenolic compound present in the myrtle extracts by the endogenous vita$\min \mathrm{E}$.

However, the effect of EEVOO extracts on AAPH-induced phospholipid oxidation was in contrast with that being predicted. Results showed that phospholipid stability was increased slightly. The protective effect for McPC-EEVOO extracts (MAE and CE) was increased from $77.2 \pm 1.1$ (EVOO control) to $79.1 \pm 1.4$ and $80.2 \pm 1.3 \%$, respectively and corresponding to an increase in the bilayer stability of 2.56 and $3.99 \%$ for the above McPCs, respectively, compared to the control. Alpha-tocopherol and BHT did not increase the phospholipid/BS aqueous dispersion stability compared to the control $(p>0.05)$. These findings were not consistent with the antioxidant activity of myrtle extract evaluated alone in AAPH system and also with the cooperative effects found for EEVOO phenolic compounds obtained in $\mathrm{Fe}^{+3} /$ AsA system. These findings suppose that the type of interaction occurring in a mixture of antioxidants was depending on the assay conditions, particularly the type of oxidant used in our experiment. An earlier work (Meyer, Heinonen, \& Frankel, 1998) showed that the combination of ellagic acid and catechin exerted antagonistic effect on LDL oxidation and this may be due to hydrogen bonding between carbonyls in ellagic acid and ortho-dihydroxyl groups in catechin which block the hydrogen donating ability of catechin. In fact, it can be supposed that there are antagonistic interactions between the EVOO and myrtle phenolic compounds that could block some hydroxyl groups other that of catechol group and thus affect the scavenging capacity of free radicals derived from AAPH decomposition and lead to decrease the effectiveness of myrtle extracts in EEVOO during phospholipid oxidation induced by AAPH.

\section{Conclusion}

In this present study, we showed that the enrichment of EVOO by myrtle extracts improved the antioxidant activity of the final product by increasing the scavenging effect toward free radicals and inhibiting the phospholipid peroxidation more effectively in $\mathrm{Fe}^{+3} /$ AsA system than in AAPH system. The enriched oil had a new composition of phenolic compounds from myrtle extracts, better antioxidant properties and may act against free radical attacks occurring during lipid digestion through several mechanisms of action. However, various kinds of interactions between antioxidants in a complex mixture may occur and thus can affect the total antioxidant capacity of the mixture. For this, it is important to choose the best combination of antioxidants when designing functional foods. Further studies are needed to evaluate the bioabsorption, bioavailability and interactions between these compounds present in myrtle enriched-EVOO, after consumption.

\section{References}

Aidi Wannes, W., Mhamdi, B., Sriti, J., Ben Jemia, M., Ouchikh, O., Hamdaoui, G., Marzouk, B. (2010). Antioxidant activities of the essential oils and methanol extracts from myrtle (Myrtus communis var. L.) leaf, stem and flower. Food and Chemical Toxicology, 48(5), 1362-1370.

Bondet, V., Cuvelier, M.-E., \& Berset, C. (2000). Behavior of phenolic antioxidants in a partitioned medium: Focus on linoleic acid peroxidation induced by iron/ ascorbic acid system. Journal of the American Oil Chemists' Society, 77(8), 813-819.

Bouaziz, M., Fki, I., Jemai, H., Ayadi, M., \& Sayadi, S. (2008). Effect of storage on refined and husk olive oils composition: Stabilization by addition of natural antioxidants from Chemlali olive leaves. Food Chemistry, 108(1), 253-262.

Chiou, A., Kalogeropoulos, N., Salta, F. N., Efstathiou, P., \& Andrikopoulos, N. K. (2009). Pan-frying of French fries in three different edible oils enriched with olive leaf extract: Oxidative stability and fate of microconstituents. LWT - Food Science and Technology, 42(6), 1090-1097.

Dairi, S., Galeano-Díaz, T., Acedo-Valenzuela, M. I., Godoy-Caballero, M. P., Dahmoune, F., Remini, H., \& Madani, K. (2015). Monitoring oxidative stability and phenolic compounds composition of myrtle-enriched extra virgin olive during heating treatment by flame, oven and microwave using reversed phase dispersive liquid-liquid microextraction (RP-DLLME)-HPLC-DAD-FLD method. Industrial Crops and Products, 65, 303-314.

Dairi, S., Madani, K., Aoun, M., Him, J. L., Bron, P., Lauret, C., ... Carbonneau, M. A. (2014). Antioxidative properties and ability of phenolic compounds of Myrtus communis leaves to counteract in vitro LDL and phospholipid aqueous dispersion oxidation. Journal of Food Science, 79(7), C1260-1270.

Dudonne, S., Vitrac, X., Coutiere, P., Woillez, M., \& Merillon, J.-M. (2009) Comparative study of antioxidant properties and total phenolic content of 30 plant extracts of industrial interest using DPPH, ABTS, FRAP, SOD, and ORAC assays. Journal of Agricultural and Food Chemistry, 57(5), 1768-1774.

Gonçalves, S., Gomes, D., Costa, P., \& Romano, A. (2013). The phenolic content and antioxidant activity of infusions from Mediterranean medicinal plants. Industrial Crops and Products, 43, 465-471.

Gordon, M. H., \& Roedig-Penman, A. (1998). Antioxidant activity of quercetin and myricetin in liposomes. Chemistry and Physics of Lipids, 97(1), 79-85.

Goupy, P., Vulcain, E., Caris-Veyrat, C., \& Dangles, O. (2007). Dietary antioxidants as inhibitors of the heme-induced peroxidation of linoleic acid: Mechanism of action and synergism. Free Radical Biology and Medicine, 43(6), 933-946.

Gutiérrez, M. E., Garcia, A. F., Africa de Madariaga, M., Sagrista, M. L., Casadó, F. J., \& Mora, M. (2003). Interaction of tocopherols and phenolic compounds with membrane lipid components: Evaluation of their antioxidant activity in a liposomal model system. Life Sciences, 72(21), 2337-2360.

Gutierrez, V. R., de la Puerta, R., \& Catalá, A. (2001). The effect of tyrosol, hydroxytyrosol and oleuropein on the non-enzymatic lipid peroxidation of rat liver microsomes. Molecular and Cellular Biochemistry, 217(1-2), 35-41.

Hidalgo, M., Sánchez-Moreno, C., \& de Pascual-Teresa, S. (2010). Flavonoidflavonoid interaction and its effect on their antioxidant activity. Food Chemistry, 121(3), 691-696.

Ishimoto, H., Tai, A., Yoshimura, M., Amakura, Y., Yoshida, T., Hatano, T., \& Ito, H. (2011). Antioxidative properties of functional polyphenols and their metabolites assessed by an ORAC assay. Bioscience, Biotechnology, and Biochemistry, 76(2), 395-399. 
Kalantzakis, G., Blekas, G., Pegklidou, K., \& Boskou, D. (2006). Stability and radicalscavenging activity of heated olive oil and other vegetable oils. European Journal of Lipid Science and Technology, 108(4), 329-335.

Kenmogne-Domguia, H. B., Moisan, S., Viau, M., Genot, C., \& Meynier, A. (2014). The initial characteristics of marine oil emulsions and the composition of the media inflect lipid oxidation during in vitro gastrointestinal digestion. Food Chemistry, $152,146-154$.

Lorrain, B., Dangles, O., Genot, C., \& Dufour, C. (2009). Chemical modeling of hemeinduced lipid oxidation in gastric conditions and inhibition by dietary polyphenols. Journal of Agricultural and Food Chemistry, 58(1), 676-683.

Losada Barreiro, S., Bravo-Díaz, C., Paiva-Martins, F., \& Romsted, L. S. (2013). Maxima in antioxidant distributions and efficiencies with increasing hydrophobicity of gallic acid and its alkyl esters. The pseudophase model interpretation of the "Cutoff Effect". Journal of Agricultural and Food Chemistry, 61(26), 6533-6543.

Marinova, E., Toneva, A., \& Yanishlieva, N. (2008). Synergistic antioxidant effect of $\alpha$-tocopherol and myricetin on the autoxidation of triacylglycerols of sunflower oil. Food Chemistry, 106(2), 628-633.

Messaoud, C., Laabidi, A., \& Boussaid, M. (2012). Myrtus communis L. infusions: The effect of infusion time on phytochemical composition, antioxidant, and antimicrobial activities. Journal of Food Science, 77(9), C941-C947.

Meyer, A. S., Heinonen, M., \& Frankel, E. N. (1998). Antioxidant interactions of catechin, cyanidin, caffeic acid, quercetin, and ellagic acid on human LDL oxidation. Food Chemistry, 61(1), 71-75.

Minioti, K. S., \& Georgiou, C. A. (2010). Comparison of different tests used in mapping the Greek virgin olive oil production for the determination of its total antioxidant capacity. Grasas y Aceites, 61(1).

Mladěnka, P., Macáková, K., Filipský, T., Zatloukalová, L., Jahodářr, L., Bovicelli, P., ... Saso, L. (2011). In vitro analysis of iron chelating activity of flavonoids. Journal of Inorganic Biochemistry, 105(5), 693-701.

Monde, A., Carbonneau, M.-A., Michel, F. O., Lauret, C., Diabate, S., Konan, E., ... Cristol, J.-P. (2011). Potential health implication of in vitro human low-density lipoprotein-vitamin e oxidation modulation by polyphenols derived from Côte D'ivoire's oil palm species. Journal of Agricultural and Food Chemistry, 59(17), 9166-9171.
Oboh, G., Puntel, R., \& Rocha, J. (2007). Hot pepper (Capsicum annuum, Tepin and Capsicum chinese, Habanero) prevents $\mathrm{Fe}^{2+}$-induced lipid peroxidation in brain in vitro. Food Chemistry, 102(1), 178-185.

Paiva-Martins, F., \& Gordon, M. H. (2002). Effects of pH and ferric ions on the antioxidant activity of olive polyphenols in oil-in-water emulsions. Journal of the American Oil Chemists' Society, 79(6), 571-576.

Palafox-Carlos, H., Gil-Chávez, J., Sotelo-Mundo, R. R., Namiesnik, J., Gorinstein, S., \& González-Aguilar, G. A. (2012). Antioxidant interactions between major phenolic compounds found in 'Ataulfo' mango pulp: Chlorogenic, gallic. Protocatechuic and vanillic acids. Molecules, 17(11), 12657-12664.

Proestos, C., \& Komaitis, M. (2008). Application of microwave-assisted extraction to the fast extraction of plant phenolic compounds. LWT - Food Science and Technology, 41(4), 652-659.

Romani, A., Campo, M., \& Pinelli, P. (2012). HPLC/DAD/ESI-MS analyses and antiradical activity of hydrolyzable tannins from different vegetal species. Food Chemistry, 130(1), 214-221.

Romani, A., Pinelli, P., Mulinacci, N., Vincieri, F., \& Tattini, M. (1999). Identification and quantitation of polyphenols in leaves of Myrtus communis L. Chromatographia, 49(1-2), 17-20.

Rosenblat, M., Volkova, N., Coleman, R., Almagor, Y., \& Aviram, M. (2008). Antiatherogenicity of extra virgin olive oil and its enrichment with green tea polyphenols in the atherosclerotic apolipoprotein-E-deficient mice: Enhanced macrophage cholesterol efflux. The Journal of Nutritional Biochemistry, 19(8), 514-523.

Saija, A., Trombetta, D., Tomaino, A., Lo Cascio, R., Princi, P., Uccella, N., ... Castelli, F. (1998). In vitro'evaluation of the antioxidant activity and biomembrane interaction of the plant phenols oleuropein and hydroxytyrosol. International Journal of Pharmaceutics, 166(2), 123-133.

Suárez, M., Romero, M. P., Ramo, T., \& Motilva, M. J. (2011). Stability of a phenolenriched olive oil during storage. European Journal of Lipid Science and Technology, 113(7), 894-903.

Yuji, H., Weiss, J., Villeneuve, P., López Giraldo, L. J., Figueroa-Espinoza, M.-C., \& Decker, E. A. (2007). Ability of surface-active antioxidants to inhibit lipid oxidation in oil-in-water emulsion. Journal of Agricultural and Food Chemistry, 55 (26), 11052-11056. 